\title{
Serum CC chemokine ligand-18 predicts lung disease worsening in systemic sclerosis
}

\author{
K.P. Tiev*, T. Hua-Huy , A. Kettaneh*, M. Gain*, S. Duong-Quy\#, C. Tolédano*, \\ J. Cabane* and A.T. Dinh-Xuan\#
}

ABSTRACT: Elevated serum $C C$ chemokine ligand $(C C L) 18$ reflects lung fibrosis activity in systemic sclerosis (SSc) and could be an early marker of lung function worsening. Therefore, we sought to evaluate whether serum CCL18 levels at baseline could predict worsening of lung disease in SSc.

In this prospective study, 83 SSc patients were analysed longitudinally over a 4-yr observation period for the risk of occurrence of combined deleterious events, defined as a $10 \%$ decrease from baseline of total lung capacity or forced vital capacity \% predicted, or death, according to serum CCL18 at inclusion. Receiver operating characteristic (ROC) curve analysis was performed for prediction of events during the first year after inclusion.

The best cut-off level of serum CCL18 for prediction of a combined event within the follow-up period was $187 \mathrm{ng} \cdot \mathrm{mL}^{-1}$, with $53 \%$ sensitivity and $96 \%$ specificity (area under the ROC curve 0.86 ; $\mathbf{p}<\mathbf{0 . 0 0 1 )}$. After a mean \pm SD follow-up of $33.7 \pm 10.8$ months, a higher rate of disease progression occurred in the group with serum CCL18 levels $>187 \mathrm{ng} \cdot \mathrm{mL}^{-1}$. The adjusted hazard ratio was 5.36 (95\% Cl 2.44-11.75; $p<0.001)$.

In summary, serum CCL18 is an accurate predictive biomarker for the identification of patients with a higher risk of subsequent scleroderma lung disease worsening.

KEYWORDS: Chemokines, interstitial lung disease, prognosis, systemic sclerosis

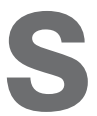
ystemic sclerosis (SSc) is a chronic autoimmune connective tissue disease characterised by sclerotic changes in skin and internal organs, with poor prognosis. Interstitial lung disease (ILD) occurs in $>50 \%$ of SSc patients and is the leading cause of death [1]. It remains a great challenge to develop biomarkers that could differentiate SSc patients who will have progressive disease from those having slow or stable disease in order to give appropriate treatment and follow-up care.

CC chemokine ligand (CCL)18 is a chemokine secreted mainly by alveolar macrophages (M2 phenotype) [2] alternatively activated by T-helper cell type 2 cytokines and plays an important role in the immune-mediated lung fibrosis processes in idiopathic pulmonary fibrosis (IPF) [3] and scleroderma lung disease [3,4]. A high amount of CCL18 produced by alveolar inflammatory cells [5] could induce an overproduction of collagen by lung fibroblasts through Sp1 signalling and basal
Smad3 activity, independently of autocrine transforming growth factor- $\beta[6,7]$.

In ILD associated with SSc, although several data have strongly suggested that CCL18 reflected an alveolar inflammatory activity, leading to lung fibrosis and subsequent worsening of lung function. Serum CCL18 fulfilled criteria for a potential biomarker of lung fibrosis activity in ILD associated with SSc [3, 4]. Its prognostic value on lung function worsening over time in patients with SSc has not yet been prospectively assessed in a longterm follow-up study. We hypothesised that elevated serum CCL18 level, reflecting the underlying sustained lung inflammatory activity, could herald the subsequent lung function worsening in SSc. We aimed to determine whether the serum CCL18 concentration could provide truly valuable information to identify those SSc patients at higher risk of having subsequent lung function worsening over time in a prospective cohort study.
AFFILIATIONS

*University of Paris VI, Dept of Internal Medicine, St Antoine Hospital, and

\#University of Paris V, Dept of Physiology, Cochin Hospital, Paris, France.

CORRESPONDENCE

A.T. Dinh-Xuan

Université Paris V

Dept of Physiology

Cochin Hospital

27 rue du Faubourg Saint-Jacques

75679 Paris cedex 14

France

E-mail: anh-tuan.dinh-xuan@

cch.aphp.fr

Received:

Jan 112011

Accepted after revision:

April 292011

First published online:

July 202011 


\section{METHODS}

\section{Patients}

Study design

The inclusion period of the cohort study was from November 2004 to November 2007, and was conducted in the Dept of Internal Medicine, Hôpital Saint-Antoine (Paris, France). The follow-up of this time-to-event-driven study ended in June 2010. Patients eligible for the study were those who met the following criteria.

\section{Inclusion criteria}

Patients were considered for inclusion if they were $>18$ yrs of age, and had a diagnosis of SSc and its subsets according to the American College of Rheumatology criteria [8] and those of LERoY et al. [9], respectively. Patients with SSc were eligible irrespective of their forced vital capacity (FVC) or diffusing capacity of the lung for carbon monoxide (DL,CO). Patients with ILD, as diagnosed by high-resolution computed tomography (HRCT), and patients treated with immunosuppressive therapy were also eligible.

\section{Exclusion criteria}

Exclusion criteria were: the presence of recent upper airway tract infection, pneumonia, or other systemic infection in the 3 months prior to the study; and IPF or ILD associated with connective tissue diseases other than SSc.

\section{Data collection}

\section{Baseline}

The study was approved by the local ethics committee (Comités de Protection des Personnes "Ile-de-France V", Hôpital SaintAntoine). After giving written informed consent, patients routinely underwent lung HRCT, echocardiogram and pulmonary function tests (PFTs) [10]. ILD was considered present if pulmonary HRCT demonstrated compatible changes in reticular or airspace opacities according to the American Thoracic Society (ATS)/European Respiratory Society (ERS) consensus classification [11]. Serum samples were frozen at $-80^{\circ} \mathrm{C}$ until serum CCL18 measurement by ELISA.

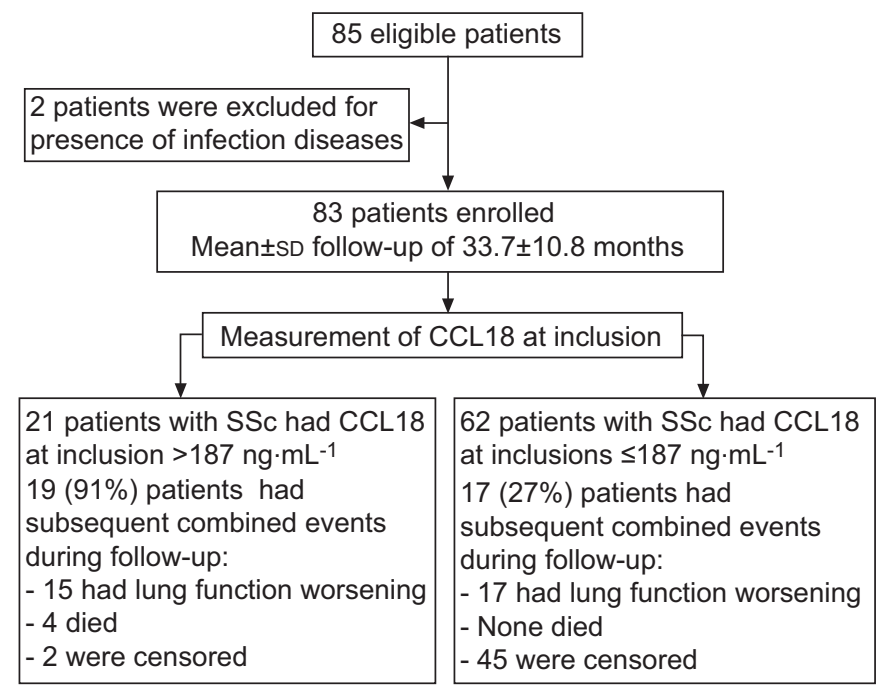

FIGURE 1. Study population. CCL: CC chemokine ligand; SSc: systemic sclerosis.
Follow-up

Survival data were obtained from visits and telephone interviews. The changes in PFTs were determined by calculating percentage differences in PFT measurements between baseline at the time of the visit when CCL18 was measured in the serum and every year or earlier, when clinically indicated, over a 4-yr period. A decrease of $>10 \%$ predicted in total lung capacity (TLC) or FVC was considered significant according to the ATS/ERS criteria [11] and a previous report [12]. Patients who had not been seen within 6 months were called to confirm their vitality. Serum CCL18 measurements were also performed at the first following visit.

\section{Measurement of serum CCL18}

CCL18 was quantified using a DuoSet ELISA development kit (R\&D Systems Europe, Abingdon, UK). All serum samples were diluted to 1:200 and measured in duplicate. Intra-assay coefficients of variation of $<10 \%$ were accepted. To ensure the reliability of measurement, we assayed CCL18 from 16 healthy volunteers as controls.

\section{Statistical analysis}

First, we assessed the optimal threshold and the diagnostic performance of CCL18 for identifying SSc patients who were going to have subsequent lung disease worsening or to die within the 2 yrs after inclusion (positive if a combined event occurred within 2 yrs of follow-up and negative if a combined event did not occur) by using receiver operating characteristic (ROC) curve analysis.

Secondly, SSc patients were equally categorised into two groups, patients with a serum CCL18 level higher than optimal threshold and other patients. Characteristics for the group of SSc patients with serum CCL18 equal to or lesser than optimal cut-off versus those with greater levels of serum CCL18 were compared using unpaired t-tests for continuous parameters and Chi-squared tests for categorical measures. Continuous and categorical values are presented as mean $\pm \mathrm{SD}$ and $\%$, respectively. A p-value of $<0.05$ was considered statistically significant.

To estimate the predictive value of serum CCL18 on lung function outcomes during the whole follow-up period, cumulative risks were computed by Kaplan-Meier analysis. We used Cox proportional hazards models, and full backward and forward multivariate analysis to assess the risk of developing worse lung function over time for patients whose CCL18 was higher than the best threshold, and compared this risk with that of the remaining patients.

Next, we assessed the relationship between change in FVC and variation of serum CCL18 level between baseline and the last visit by a linear regression model.

\section{RESULTS}

\section{Characteristics of population at baseline}

85 patients with SSc were eligible in this prospective study. Two patients were excluded for pneumonia and sigmoiditis (fig. 1). 83 patients were consecutively included in the study (table 1). After a mean \pm SD follow-up of $33.7 \pm 10.8$ months, four SSc patients died and no patient was lost to follow-up. Composite events, defined as death or $10 \%$ decrease in TLC or FVC, occurred in $43 \%$ of patients. 
TABLE 1 Baseline characteristics of patients

\begin{tabular}{|c|c|c|c|c|}
\hline & All SSc patients & $\mathrm{CCL} 18>187 \mathrm{ng} \cdot \mathrm{mL}^{-1}$ & $\mathrm{CCL} 18 \leqslant 187 \mathrm{ng} \cdot \mathrm{mL}^{-1}$ & $\mathrm{p}$-value \\
\hline Subjects & 83 & 21 & 62 & \\
\hline Females & $73(88.0)$ & $16(76.6)$ & 57 (91.9) & 0.13 \\
\hline Duration of disease yrs & $11.6 \pm 11.1$ & $11.1 \pm 9.8$ & $11.6 \pm 11.5$ & 0.84 \\
\hline Diffuse & $35(42.2)$ & $15(71.4)$ & $20(32.3)$ & 0.004 \\
\hline Antitopoisomerase I antibody & $37(44.6)$ & $15(71.4)$ & $22(35.5)$ & 0.004 \\
\hline History of smoking & 19 (22.9) & $5(23.8)$ & $14(22.5)$ & 0.85 \\
\hline ILD & $46(55.4)$ & $18(85.7)$ & $28(45.2)$ & 0.003 \\
\hline FVC \% pred & $93 \pm 20$ & $84 \pm 16$ & $96 \pm 21$ & 0.01 \\
\hline FEV $1 \%$ pred & $90 \pm 17$ & $82 \pm 15$ & $93 \pm 19$ & 0.02 \\
\hline$D \mathrm{~L}, \mathrm{co} \%$ pred & $62 \pm 16$ & $50 \pm 10$ & $65 \pm 16$ & $<0.001$ \\
\hline$>40 \mathrm{mmHg}$ & $13(15.6)$ & $9(42.9)$ & $4(6.4)$ & 0.001 \\
\hline Immunosuppressive treatment & $15(18.1)$ & $6(28.6)$ & $9(14.5)$ & 0.26 \\
\hline
\end{tabular}

Data are presented as $n$, mean \pm SD or $n(\%)$, unless otherwise stated. SSc: systemic sclerosis; CCL: CC chemokine ligand; ILD: interstitial lung disease; FVC: forced vital capacity; \% pred: \% predicted; FEV1: forced expiratory volume in $1 \mathrm{~s}$; DL,CO: diffusing capacity of the lung for carbon monoxide; Ppa: pulmonary artery pressure.

\#: assessed by echocardiogram.

\section{Threshold for identifying SSc patients who were going to have subsequent lung disease worsening or die during the 2 yrs after inclusion}

The baseline serum levels ranged $33-649 \mathrm{ng} \cdot \mathrm{mL}^{-1}$ and their distribution in the whole studied population is shown in figure 2. The best cut-off for identifying subsequent lung disease worsening within 2 yrs was $187 \mathrm{ng} \cdot \mathrm{mL}^{-1}$ (95\% CI 159$\left.218 \mathrm{ng} \cdot \mathrm{mL}^{-1}\right)$ with an area under the ROC curve of $0.86(95 \%$ CI $0.78-0.93)(\mathrm{p}<0.001)$, and sensitivity and specificity of 0.53 (95\% CI $0.35-0.70)$ and 0.96 (95\% CI $0.85-0.99)$, respectively (fig. 2). The internal validation of this CCL18 cut-off is provided as supplementary data available online (table S1).

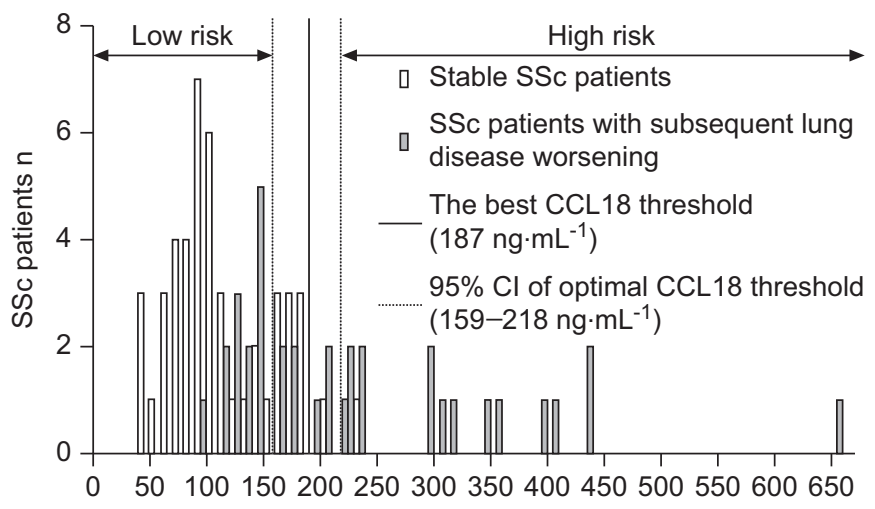

Baseline CCL18 level ng. $\mathrm{mL}^{-1}$

FIGURE 2. Distribution of baseline serum CC chemokine ligand (CCL)18 serum level in patients with systemic sclerosis (SSc).
Characteristics of population at baseline according to the best CCL18 threshold for predicting subsequent combined events during the first 2 yrs after inclusion

We used the best CCL18 cut-off $\left(187 \mathrm{ng} \cdot \mathrm{mL}^{-1}\right)$ to stratify the study population. Composite events, defined as death, or a $10 \%$ decrease in TLC or FVC, occurred in 91\% of patients with CCL18 $>187 \mathrm{ng} \cdot \mathrm{mL}^{-1}$ and $27 \%$ of those with CCL18 $\leqslant 187 \mathrm{ng} \cdot \mathrm{mL}^{-1}$. There were significantly higher percentages of the diffuse form of the disease $(p=0.004)$, presence of antitopoisomerase I antibodies $(p=0.004)$, ILD ( $p=0.003)$, systolic pulmonary artery pressure $>40 \mathrm{mmHg}(\mathrm{p}=0.001)$, and lower FVC $(\mathrm{p}=0.01)$ or $D \mathrm{~L}, \mathrm{CO}(\mathrm{p}<0.001)$ in the group of SSc patients with high levels of CCL18, as compared with those with a low level of CCL18. Immunosuppressive therapy, including corticosteroids and immunosuppressive agents, was comparable between the two groups. Further cross-sectional analysis of the baseline data is provided in the online supplementary material.

\section{Association between baseline serum CCL18 level and lung function worsening over time}

Progression of lung function decline differed between SSc patients with high and low serum CCL18 levels at baseline. Times to $25 \%$ events were significantly shorter in patients with high CCL18 levels (22 months, 95\% CI 14.0-23.5 months), as compared with those with low CCL18 levels (37 months, 95\% CI 28.7-37.4 months) (log-rank $\mathrm{p}<0.001$ ) (fig. 3).

In a univariate Cox proportional hazards model, serum baseline CCL18 level $>187 \mathrm{ng} \cdot \mathrm{mL}^{-1}$ was associated with a higher risk of lung function worsening over time (hazard ratio (HR) 5.32, 95\% CI 2.70-10.52; $\mathrm{p}<0.001$; table 2). Linear regression analysis using CCL18 as a continuous variable to predict subsequent change in 


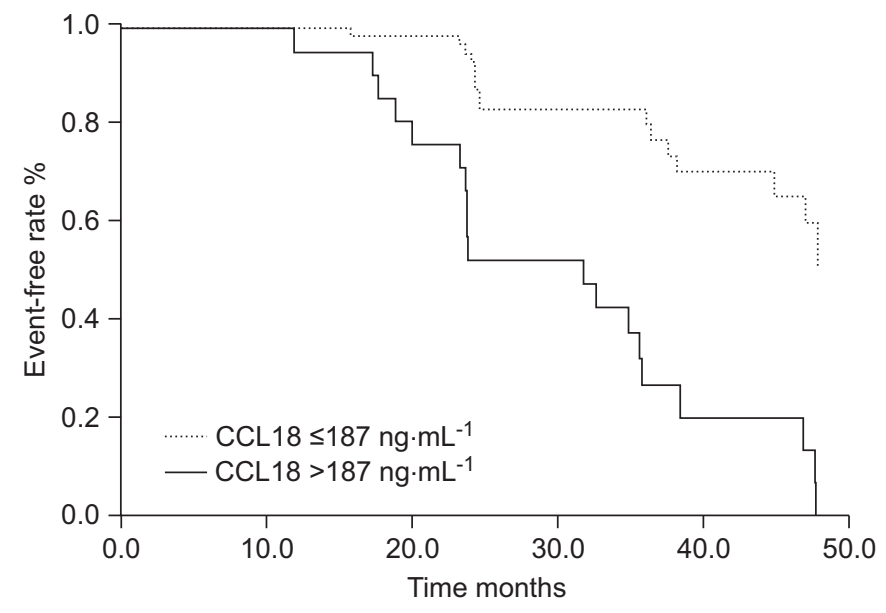

FIGURE 3. Kaplan-Meier analysis grouped by baseline serum CC chemokine ligand (CCL) 18 level. Log-rank $p<0.001$.

FVC was consistent with the Cox proportional hazards model and is available in the online supplementary material (table S2).

In multivariate analyses, when large demographic data, characteristics of disease, serum inflammatory markers and immunosuppressive therapy, were put into the backward and forward multivariate Cox proportional hazards models, serum CCL18 concentration at baseline remained an accurate predictive factor of the subsequent lung disease worsening (HR 5.36, 95\% CI 2.44-11.75; $p<0.001$; table 3). The baseline CCL18 levels of four SSc patients who died during the follow-up period were 139, 228, 230 and $649 \mathrm{ng} \cdot \mathrm{mL}^{-1}$.

\begin{tabular}{|c|c|c|}
\hline & HR (95\% Cl) & p-value \\
\hline $\mathrm{CCL} 18>187 \mathrm{ng} \cdot \mathrm{mL}^{-1}$ & $5.32(2.70-10.52)$ & $<0.001$ \\
\hline $\operatorname{Age}^{\#, \oplus}$ & $1.02(0.98-1.05)$ & 0.19 \\
\hline Female & $0.53(0.24-1.20)$ & 0.11 \\
\hline Diffuse form of SSc & $2.09(1.08-4.05)$ & 0.03 \\
\hline Anticentromere antibody & $0.61(0.31-1.18)$ & 0.14 \\
\hline Antitopoisomerase I antibody & $1.62(0.84-3.13)$ & 0.15 \\
\hline History of smoking & $0.81(0.35-1.85)$ & 0.62 \\
\hline Duration of disease ${ }^{\#, 9}$ & $0.99(0.95-1.02)$ & 0.46 \\
\hline Presence of ILD & $2.40(1.15-4.99)$ & 0.02 \\
\hline$D \mathrm{~L}, \mathrm{CO}^{\#,+}$ & $0.96(0.94-0.98)$ & 0.001 \\
\hline $\mathrm{FVC}^{\#,+}$ & $0.99(0.97-1.00)$ & 0.11 \\
\hline Systolic $P \mathrm{pa}^{\S}>40 \mathrm{mmHg}$ & $3.19(1.55-6.56)$ & 0.002 \\
\hline Fibrinogen $\mathbf{n}^{\#, f}$ & $1.41(1.00-2.00)$ & 0.05 \\
\hline Immunosuppressive treatment & $2.18(1.04-4.56)$ & 0.04 \\
\hline
\end{tabular}

HR: hazard ratio; CCL: CC chemokine ligand; SSc: systemic sclerosis; ILD: interstitial lung disease; $D\llcorner, \mathrm{CO}$ : diffusing capacity of the lung for carbon monoxide; FVC: forced vital capacity; Ppa: pulmonary artery pressure. ${ }^{\# \text { : as a }}$ continuous variable; ${ }^{\bullet}$ : per $\mathrm{yr}^{+}{ }^{+}$: per $\%$ predicted; ${ }^{\text {s. }}$ : assessed by echocardiogram; ${ }^{f}$ : per $g \cdot \mathrm{L}^{-1}$.

\begin{tabular}{llc} 
TABLE 3 & $\begin{array}{l}\text { Risks of lung function worsening over time in } \\
\text { patients with systemic sclerosis (SSC) according } \\
\text { to optimal CC chemokine ligand (CCL) 18 cut-off }\end{array}$ \\
& HR (95\%Cl) & p-value \\
\hline CCL18 $>\mathbf{1 8 7} \mathbf{~} \mathbf{g} \cdot \mathbf{~ m L}^{-1}$ & $5.36(2.44-11.75)$ & $<0.001$ \\
Duration of disease & $0.97(0.93-1.01)$ & 0.10 \\
DL,Co* & $0.97(0.95-1.00)$ & 0.04 \\
\hline
\end{tabular}

Full backward analysis provided a final model that we also obtained using the forward multivariate analysis. The initial multivariate analysis model was adjusted for age and duration of disease, and all other parameters with a $\mathrm{p}$ value of $<0.20$ in the univariate analysis (i.e. sex, form of SSc, presence of interstitial lung disease, forced vital capacity, presence of systolic pulmonary artery pressure of $\geqslant 40 \mathrm{mmHg}$, fibrinogen, immunosuppressive therapy and forced vital capacity). HR: hazard ratio; $D \mathrm{~L}, \mathrm{CO}$ : diffusing capacity of the lung for carbon monoxide. ${ }^{\#}$ : per yr; ": per \% predicted.

\section{Relationship between variation of serum CCL18 level and change in FVC}

After adjustment for FVC at baseline, FVC at the follow-up visit was related to CCL18 at baseline but not to its change (serum CCL18 at inclusion minus that at the follow-up visit) (table 4). Serum levels from SSc patients with CCL18 higher than the optimal threshold remained $>187 \mathrm{ng} \cdot \mathrm{mL}^{-1}$ during follow-up (fig. 4).

\section{DISCUSSION}

In this present prospective cohort study, we found, for the first time, that a baseline serum CCL18 level $>187 \mathrm{mg} \cdot \mathrm{mL}^{-1}$ was a strong independent predictive factor for subsequent lung disease worsening in patients with SSc (HR 5.36). CCL18 provided additional information that helped us to identify SSc patients with progressive lung disease.

In SSc, there are few relevant biomarkers that allow us to predict progression of lung fibrosis or survival [13]. Currently, several biological markers, such as neutrophilic alveolitis surfactant A and D [14], or cytokines, such as tumour necrosis factor- $\alpha$ [15], and chemokines, such as interleukin-8 [16] and CCL2 [16-18], and alveolar concentration of nitric oxide [19, 20], have been shown to be associated with the severity of ILD in SSc. Nevertheless, for each biomarker, long-term follow-up

\begin{tabular}{|c|c|c|c|}
\hline \multirow[t]{2}{*}{ TABLE 4} & \multicolumn{3}{|c|}{$\begin{array}{l}\text { Forced vital capacity (FVC) at follow-up visit } \\
\text { according CC chemokine ligand (CCL) } 18 \text { at } \\
\text { baseline, and its variation between inclusion and } \\
\text { the following visit }\end{array}$} \\
\hline & & $\boldsymbol{\beta} \pm \mathrm{SE}$ & p-value \\
\hline \multicolumn{2}{|l|}{ Intercept } & $9.417 \pm 7.057$ & 0.19 \\
\hline \multicolumn{2}{|c|}{ Baseline FVC $\#$} & $0.952 \pm 0.061$ & $<0.0001$ \\
\hline \multicolumn{2}{|c|}{ Baseline serum CCL18 level" } & $-0.035 \pm 0.014$ & 0.01 \\
\hline \multicolumn{2}{|c|}{ Variation of CCL18 levels ${ }^{\top,+}$} & $-0.016 \pm 0.012$ & 0.16 \\
\hline
\end{tabular}




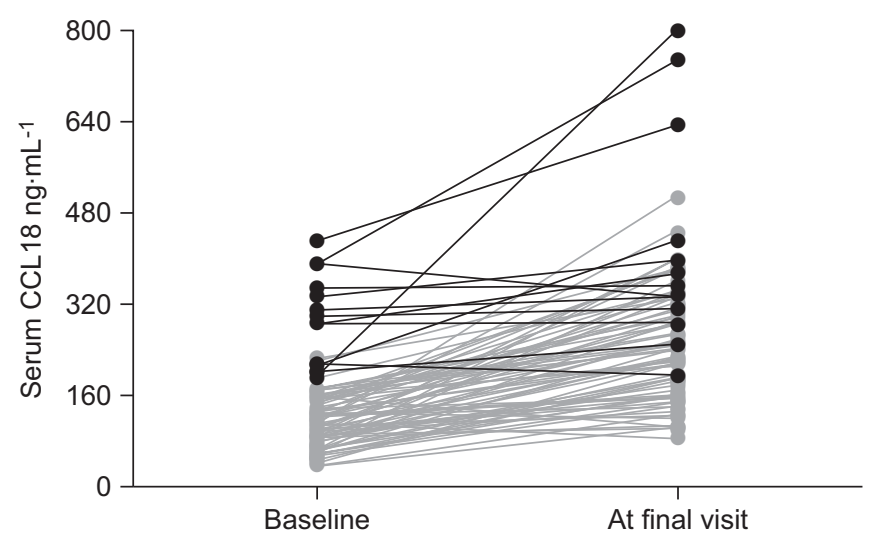

FIGURE 4. Change in CC chemokine ligand (CCL)18 levels during follow-up. Black lines represents systemic sclerosis patients with baseline serum CCL18 levels above the best threshold $\left(187 \mathrm{ng} \cdot \mathrm{mL}^{-1}\right)$ and grey lines represent those with baseline serum CCL18 levels $\leqslant 187 \mathrm{ng} \cdot \mathrm{mL}^{-1}$

studies showing relevant prognostic value for progression are lacking. To date, FVC $[12,21]$ and $D \mathrm{~L}, \mathrm{CO}[22]$ remain the most relevant predictive factors in ILD. In order to assess scleroderma lung disease, which has become the leading cause of death in SSc, several physiological lung parameters, such as FVC and $\mathrm{DL}, \mathrm{CO}$, have been used as surrogate markers for mortality or impairment of lung function in clinical trial [23, 24]. Decreased FVC reflected the severity of lung fibrosis and FVC $<55 \%$ pred increased 9-yr mortality in the diffuse form of SSc [25]. Low $D \mathrm{~L}, \mathrm{CO}$ was also a predictor for the occurrence of pulmonary arterial hypertension [26] and mortality [27]. However, patients with more severe impairment of FVC or DL,CO were more likely to have worsening lung function than others. Unlike physiological lung parameters, biological markers have the advantage of describing the underlying biological activity leading to lung fibrosis independently of the subjectivity of patient-related symptoms and fibrosis-related mechanical changes of the lung parenchyma. In the present study, serum level of CCL18 seems to satisfy these requirements of a good biomarker.

Previous studies have shown that high CCL18 levels were associated with increased profibrotic activity in scleroderma lung disease and high serum levels could reflect progressive lung disease [3]. Consistent with previous findings, we found relationships between elevated CCL18, and severity of scleroderma lung disease, extent of skin fibrosis and systolic pulmonary pressure $>40 \mathrm{mmHg}$ estimated by echocardiogram.

Recently, in IPF, PRASSE et al. [28] reported that serum CCL18 had an accurate prognostic value on disease-related death (HR 8). Although previous studies have proposed a potential link between CCL18 and SSc-ILD, we present here the first longterm follow-up data showing that serum CCL18 level predicted lung function decline over time or death. In a large cohort study, SIN et al. [29] reported that CCL18 levels were significantly related to age, sex, body mass index and smoking status. As the SSc patients in that study were predominantly female and their mean age was $\sim 55$ yrs, elevated CCL18 could be biased by age and sex. So, we took into account age, sex, body mass index and smoking status in the multivariate analysis. Serum CCL18 level was still an accurate predictive marker for lung function decline over time or death (HR 5.36) after adjustment for factors including duration of disease, baseline FVC and DL,CO.

The rate of the progressive form of SSc in the present study was higher than that reported in previous large cohort studies $[21,30]$. Compared with previous studies, patients from the present study seemed to have more severe disease, as evidenced by the presence of severe organ involvement in most patients and four deaths during the first year after inclusion ( $4.8 \%$ of subjects). The fact that our hospital is a national reference centre receiving the most severe cases of SSc might account for the high rate of combined outcomes.

We showed the robustness of an optimal CCL18 threshold for prediction of lung disease worsening in SSc by internal validation. However, external validation by an independent cohort is needed prior to recommending intervention studies targeting CCL18.

The variation of serum CCL18 obtained by repeated measurement did not provide more information on change in FVC during history of progression of lung disease. However, the fact that serum levels of patients with CCL18 higher than the optimal cut-off still remained higher during follow-up may reflect a continuous deterioration of lung function, even in those patients in whom loss of lung volume due to lung fibrosis had already begun.

Our findings showed that serum CCL18 had a good predictive value for worsening of lung disease in patients with SSc and it might therefore be used as a surrogate biomarker to identify atrisk SSc patients. Further studies are needed to show whether therapies may have an influence on CCL18 levels.

\section{SUPPORT STATEMENT}

This study was supported by the Legs Poix (Chancellerie des Universités de Paris, Paris, France) and the Association des Sclérodermiques de France (Sorel-Moussel, France).

\section{STATEMENT OF INTEREST}

None declared.

\section{REFERENCES}

1 Steen VD, Medsger TA. Changes in causes of death in systemic sclerosis, 1972-2002. Ann Rheum Dis 2007; 66: 940-944.

2 Schutyser E, Richmond A, Van Damme J. Involvement of CC chemokine ligand 18 (CCL18) in normal and pathological processes. J Leukoc Biol 2005; 78: 14-26.

3 Prasse A, Pechkovsky DV, Toews GB, et al. CCL18 as an indicator of pulmonary fibrotic activity in idiopathic interstitial pneumonias and systemic sclerosis. Arthritis Rheum 2007; 56: 1685-1693.

4 Kodera M, Hasegawa M, Komura K, et al. Serum pulmonary and activation-regulated chemokine/CCL18 levels in patients with systemic sclerosis: a sensitive indicator of active pulmonary fibrosis. Arthritis Rheum 2005; 52: 2889-2896.

5 Luzina IG, Atamas SP, Wise R, et al. Gene expression in bronchoalveolar lavage cells from scleroderma patients. Am J Respir Cell Mol Biol 2002; 26: 549-557.

6 Atamas SP, Luzina IG, Choi J, et al. Pulmonary and activationregulated chemokine stimulates collagen production in lung fibroblasts. Am J Respir Cell Mol Biol 2003; 29: 743-749. 
7 Luzina IG, Highsmith K, Pochetuhen K, et al. PKCalpha mediates CCL18-stimulated collagen production in pulmonary fibroblasts. Am J Respir Cell Mol Biol 2006; 35: 298-305.

8 Preliminary criteria for the classification of systemic sclerosis (scleroderma). Subcommittee for scleroderma criteria of the American Rheumatism Association Diagnostic and Therapeutic Criteria Committee. Arthritis Rheum 1980; 23: 581-590.

9 LeRoy EC, Black C, Fleischmajer R, et al. Scleroderma (systemic sclerosis): classification, subsets and pathogenesis. I Rheumatol 1988; 15: 202-205.

10 Miller MR, Hankinson J, Brusasco V, et al. Standardisation of spirometry. Eur Respir J 2005; 26: 319-338.

11 American Thoracic Society, European Respiratory Society. International Multidisciplinary Consensus Classification of the Idiopathic Interstitial Pneumonias. This joint statement of the American Thoracic Society (ATS), and the European Respiratory Society (ERS) was adopted by the ATS board of directors, June 2001 and by the ERS Executive Committee, June 2001. Am J Respir Crit Care Med 2002; 165: 277-304.

12 Steen VD, Conte C, Owens GR, et al. Severe restrictive lung disease in systemic sclerosis. Arthritis Rheum 1994; 37: 1283-1289.

13 Prasse A, Muller-Quernheim J. Non-invasive biomarkers in pulmonary fibrosis. Respirology 2009; 14: 788-795.

14 Asano Y, Ihn H, Yamane K, et al. Clinical significance of surfactant protein $\mathrm{D}$ as a serum marker for evaluating pulmonary fibrosis in patients with systemic sclerosis. Arthritis Rheum 2001; 44: 1363-1369.

15 Schmidt K, Martinez-Gamboa L, Meier S, et al. Bronchoalveoloar lavage fluid cytokines and chemokines as markers and predictors for the outcome of interstitial lung disease in systemic sclerosis patients. Arthritis Res Ther 2009; 11: R111.

16 Meloni F, Caporali R, Marone Bianco A, et al. BAL cytokine profile in different interstitial lung diseases: a focus on systemic sclerosis. Sarcoidosis Vasc Diffuse Lung Dis 2004; 21: 111-118.

17 Antonelli A, Ferri C, Fallahi P, et al. CXCL10 $(\alpha)$ and CCL2 $(\beta)$ chemokines in systemic sclerosis - a longitudinal study. Rheumatology (Oxford) 2008; 47: 45-49.

18 Fujii H, Shimada Y, Hasegawa M, et al. Serum levels of a Th1 chemoattractant IP-10 and Th2 chemoattractants, TARC and MDC, are elevated in patients with systemic sclerosis. J Dermatol Sci 2004; 35: 43-51.

19 Tiev KP, Cabane J, Aubourg F, et al. Severity of scleroderma lung disease is related to alveolar concentration of nitric oxide. Eur Respir J 2007; 30: 26-30.

20 Tiev KP, Coste J, Ziani M, et al. Diagnostic value of exhaled nitric oxide to detect interstitial lung disease in systemic sclerosis. Sarcoidosis Vasc Diffuse Lung Dis 2009; 26: 32-38.

21 Goh NS, Desai SR, Veeraraghavan S, et al. Interstitial lung disease in systemic sclerosis: a simple staging system. Am J Respir Crit Care Med 2008; 177: 1248-1254.

22 Bouros D, Wells AU, Nicholson AG, et al. Histopathologic subsets of fibrosing alveolitis in patients with systemic sclerosis and their relationship to outcome. Am J Respir Crit Care Med 2002; 165: 1581-1586.

23 Tashkin DP, Elashoff R, Clements PJ, et al. Cyclophosphamide versus placebo in scleroderma lung disease. N Engl J Med 2006; 354: 2655-2666.

24 Tashkin DP, Elashoff R, Clements PJ, et al. Effects of 1-year treatment with cyclophosphamide on outcomes at 2 years in scleroderma lung disease. Am J Respir Crit Care Med 2007; 176: 1026-1034.

25 Steen VD, Medsger TA Jr. Severe organ involvement in systemic sclerosis with diffuse scleroderma. Arthritis Rheum 2000; 43: 2437-2444.

26 Steen VD, Graham G, Conte C, et al. Isolated diffusing capacity reduction in systemic sclerosis. Arthritis Rheum 1992; 35: 765-770.

27 Steen V, Medsger TA Jr. Predictors of isolated pulmonary hypertension in patients with systemic sclerosis and limited cutaneous involvement. Arthritis Rheum 2003; 48: 516-522.

28 Prasse A, Probst C, Bargagli E, et al. Serum CC-chemokine ligand 18 concentration predicts outcome in idiopathic pulmonary fibrosis. Am J Respir Crit Care Med 2009; 179: 717-723.

29 Sin DD, Miller B, Duvoix A, et al. Serum PARC/CCL-18 concentrations and health outcomes in chronic obstructive pulmonary disease. Am J Respir Crit Care Med 2011; 183: 1187-1192.

30 Assassi S, Sharif R, Lasky RE, et al. Predictors of interstitial lung disease in early systemic sclerosis: a prospective longitudinal study of the GENISOS cohort. Arthritis Res Ther 2010; 12: R166. 